\title{
Peroxiredoxin 5 overexpression enhances tumorigenicity and correlates with poor prognosis in gastric cancer
}

\author{
BOKYUNG KIM ${ }^{1 *}$, YEON SOO KIM ${ }^{2 *}$, HYE-MI AHN $^{1 *}$, HYO JIN LEE $^{3}$, MIN KYU JUNG ${ }^{4}$, HYUN YONG JEONG $^{3}$, \\ DONG KYU CHOI ${ }^{5}$, JUN HYEOG LEE ${ }^{1,5}$, SANG-RAE LEE ${ }^{6}$, JIN MAN KIM ${ }^{4}$ and DONG-SEOK LEE ${ }^{1}$ \\ ${ }^{1}$ School of Life Sciences and Biotechnology, BK21 Plus KNU Creative BioResearch Group, \\ Kyungpook National University, Daegu; ${ }^{2}$ Department of Internal Medicine, Daejeon St. Mary's Hospital, \\ The Catholic University, Daejeon; Departments of ${ }^{3}$ Internal Medicine and ${ }^{4}$ Pathology and Medical Science, \\ College of Medicine, Chungnam National University, Daejeon; ${ }^{5}$ New Drug Development Center, \\ Daegu-Gyeongbuk Medical Innovation Foundation (DGMIF), Daegu; ${ }^{6}$ The National Primate Research Center, \\ Korea Research Institute of Bioscience and Biotechnology (KRIBB), Ochang, Republic of Korea
}

Received December 22, 2016; Accepted April 7, 2017

DOI: 10.3892/ijo.2017.4013

\begin{abstract}
Gastric cancer is one of the leading causes of cancerrelated deaths worldwide. Despite the advanced surgical resection techniques and anticancer drugs currently available to treat early stage gastric cancer, the prognosis of patients with gastric cancer remains poor. The epithelial to mesenchymal transition (EMT) is an important process for the initiation of tumorigenesis. Recent studies suggested that reactive oxygen species (ROS) can promote cell migration and invasion. Thus, an imbalance of redox homeostasis can result in cancer cells exhibiting EMT properties. PRXs are upregulated in various tumors in the breast, bladder, lung, cervical, ovarian, prostate, esophageal, and hepatocellular. However, PRX expression and its impact on disease prognosis, patient survival rate, and EMT are rarely studied in the context of human gastric cancer. The expression of PRX5 was significantly correlated with tumor size, depth of tumor, lymphatic invasion in patients of gastric cancer. In addition, overexpression of PRX5 enhanced carcinogenicity by increasing the proliferation and invasiveness of gastric cancer cells via upregulation of Snail. Taken together, we suggest that PRX5 may be a potential factor that may contribute to poor prognosis of gastric cancer through
\end{abstract}

Correspondence to: Professor Dong-Seok Lee, School of Life Sciences and Biotechnology, College of Natural Sciences, Kyungpook National University, Daegu 41566, Republic of Korea

E-mail: lee1@knu.ac.kr

Professor Jin-Man Kim, Department of Pathology and Medical Science, College of Medicine, Chungnam National University, Daejeon 301-131, Republic of Korea

E-mail: jinmank@cnu.ac.kr

"Contributed equally

Key words: peroxiredoxin 5, gastric cancer, epithelial-mesenchymal transition, reactive oxygen species, tumorigenicity enhancing the mesenchymal phenotype. Finally, PRX5 is a putative therapeutic target and clinical strategy for various cancers overexpressing PRX5.

\section{Introduction}

Gastric cancer is one of the leading causes of cancer-related deaths worldwide. Although the incidence rate continues to decrease, approximately 723,000 patients died in 2012 due to gastric cancer (1-3). Currently, treatment of primary gastric cancer consists of metastasectomy, gastrectomy, radiofrequency ablation, and stereotactic body radiation therapy $(3,4)$. Despite the advanced surgical resection techniques and anticancer drugs currently available to treat early stage gastric cancer, the prognosis of patients with gastric cancer remains poor. This is typically due to distant metastasis such as liver or peritoneal and resistance to traditional drugs $(3,4)$. Thus, the 5-year survival rate of gastric cancer patients after surgery has been poorly. Therefore, there is a need to elucidate the mechanism of metastasis in order to develop better treatments and to improve the survival rate in patients with gastric cancer.

The epithelial to mesenchymal transition (EMT) is an important process for the initiation of tumorigenesis. This is characterized by a loss of polarity, cell-cell adhesion, and tight junctions in polarized epithelial cells. Also occurring in parallel, these cells become more mobile and invasive and exhibit more of a mesenchymal phenotype. During the progression of tumors, a variety of EMT regulators enhanced tumor formation and/or distant metastasis (5). This involves various molecular processes where transcription factors are activated, cell surface proteins are overexpressed, and cytoskeletal proteins are reorganized and overexpressed. These are characteristic changes during EMT, resulting in the molecular expression of EMT-related and mesenchymal cell markers that can be used towards the prognosis of gastric cancer (5-8).

Additionally, abnormal control of reactive oxygen species (ROS) has been implicated as a causal factor in $>250$ diseases and disorders, including cardiovascular disease, 
stroke, neurodegenerative disease, diabetes, asthma, ageing, and cancer $(9,10)$. ROS include superoxide anion and hydrogen peroxide, and are generated either by normal redox reactions under aerobic metabolic processes in the mitochondria, or through exposure to infectious and inflammatory stimuli such as smoking, alcohol, pollutants, radiation, and carcinogens (11). Recent studies have suggested that ROS can promote cell migration and invasion; thus, an imbalance of redox homeostasis can result in cancer cells exhibiting EMT properties (12). The long-term presence of even a small amount of ROS can pose a risk to cells because they can participate in pathophysiological processes such as protein and DNA damage and lipid peroxidation. Furthermore, ROS can participate in carcinogenesis at different stages such as initiation, promotion, and progression; thus, ROS are considered a key factor in tumorigenesis and can be a useful marker for the diagnosis and prognosis of various kinds of cancer $(2,12,13)$.

To counteract these damaging processes, cells can use several protective mechanisms to either repairing the various types of damage caused by ROS, such as by rescuing oxidized targets, or by eliminating ROS such as through the catalytic removal of free radicals, increasing free radical scavengers, and the removal of $\mathrm{Fe}$ and $\mathrm{Cu}(9,11-15)$. One of the most important protective mechanisms against ROS involves antioxidant enzymes such as catalase, glutathione peroxidase, and peroxiredoxins (PRXs) (15). A major common function of PRXs involves enzymatic degradation of hydrogen peroxide, organic hydroperoxides, and peroxynitrite (11). PRXs also play a key role in several cellular functions such as protein and lipid protection against oxidative injury, cell proliferation, differentiation, and apoptosis (9-12). Furthermore, recent evidence suggests that PRXs may be associated with tumor progression (16-18). Overexpression of PRXs in tumors have been suggested to be responsible for tumor progression, prognosis, and resistance to chemotherapy and radiotherapy $(19,20)$. Overall, PRXs are peroxidases containing high antioxidant efficacy and are associated with cancer development and tumorigenesis in several kinds of cancer (21-23).

Lastly, PRXs are upregulated in various tumors in the breast, bladder, lung, cervical, ovarian, prostate, esophageal, and hepatocellular (19,21-23). However, PRX expression and its impact on disease prognosis, patient survival rate, and EMT have rarely been studied in the context of human gastric cancer. Very few studies have addressed how the expression and function of all six PRXs affect human gastric cancer, disease progression or prognosis. Thus, to improve our understanding of PRXs in human gastric cancer, we investigated the expression of PRX1-5 in human gastric cancer tissues and correlated their expression with clinicopathological parameters.

\section{Materials and methods}

Tissue samples. Gastric cancer tissue samples were obtained from 210 consecutive patients who underwent elective surgery for gastric cancer at the Chungnam National University Hospital between 2000 and 2003. The patients underwent R0 resection with at least a D1 lymph node dissection. Adenocarcinomas from the patients' stomachs were isolated and histologically confirmed. The clinicopathological parameters assessed were established by the Japanese Gastric Cancer Association (24).
All patients signed informed consent for the therapy as well as for subsequent tissue studies. The experiments received prior approval by the institutional review board.

Tissue microarray construction. Paraffin blocks of the 210 human gastric cancer samples were identified on corresponding H\&E-stained sections. Areas of interest that represented invasive adenocarcinomas were identified and marked on the donor block. A 2-mm core from the area of interest was transferred to the recipient master block using the Tissue Microarrayer (Meditech Ind., Korea). Two stomach cancer cores were arrayed per specimen. In addition, four cores of normal gastric tissue were also sampled.

Immunohistochemistry. The stomach cancer tissue samples were fixed in $10 \%$ buffered formalin, and routinely processed and embedded in paraffin. Three-micrometer thick paraffin sections from the paraffin TMA blocks were used for immunohistochemistry (IHC) with the rabbit EnVision-HRP detection system (Dako, Carpinteria, CA, USA). Polyclonal rabbit antibody for PRX5 (AbFrontier, Korea) was used for IHC. All immunostaining steps were carried out at room temperature. After deparaffinization, antigen retrieval was performed with $10 \mathrm{mM}$ sodium citrate buffer ( $\mathrm{pH}$ 6.0) via a pressure cooker at full power for $4 \mathrm{~min}$. The tissue sections were then treated with $3 \%$ hydrogen peroxide for $10 \mathrm{~min}$. The primary antibody was diluted PRX5, 1:500 with background reducing diluent (Dako) and incubated for $30 \mathrm{~min}$. Slides were then incubated with the EnVision reagent for $30 \mathrm{~min}$. The slides were then sequentially incubated with DAB chromogen for $5 \mathrm{~min}$, counterstained with Meyer's hematoxylin, and mounted. Careful rinses with several changes of TBS- $0.3 \%$ Tween buffer were performed at each step. A rabbit IgG isotype control without the primary antibody was used as a negative control. Membranous, cytoplasmic, or nuclear staining was considered positive staining for PRX5 in gastric carcinoma tissues. The immunohistochemical staining was then categorized according to a scoring method. Tumors were classified into four grades based on the staining intensity: -, 0-10\%, no staining; +, 11-40\%, weak staining;,$++ 41-70 \%$, intermediate staining;,$+++ 71-100 \%$, strong staining. Cases with no staining (-) were assigned to the PRX5-negative group, whereas those with a score of weak staining (+) to strong staining $(+++)$ were assigned to the PRX5-positive group. Then, for deeper comparative analysis of PRX5 expression to related 5-year survival rate of patients in gastric cancer, tumors were categorized by high expression $(>40 \%$ staining, ++ and $+++)$ and low expression ( $<40 \%$ staining, - and + ) of PRX5.

Cell lines and cultures. Nine human gastric cancer cell lines were used in the present study. MKN-28, MKN-45, MKN-74, SNU-1, SNU-16, SNU-216, and SNU668 human gastric cancer cells were purchased from the Korean Cell Line Bank (Seoul, Korea) and were maintained in RPMI-1640 supplemented with $10 \%(\mathrm{v} / \mathrm{v})$ fetal bovine serum (FBS) according to the manufacturer's protocol.

Transfection and selection of stably expressing cells. For all cell lines, $1 \times 10^{5}$ cells were seeded onto 6-well plates. After $24 \mathrm{~h}$, the cells in each well were transfected with $2 \mu \mathrm{g}$ of pLenti6.3-PRX5 by using Effectene (Qiagen, CA, USA) 
according to the manufacturer's instructions. After $24 \mathrm{~h}$, the transfected cells were selected by supplementing $8 \mu \mathrm{g} / \mathrm{ml}$ blasticidin (Invitrogen, Carlsbad, CA, USA) into the media.

RNA isolation and reverse transcription polymerase chain reaction $(R T-P C R)$. Total RNA was isolated from SNU-216, SNU-668, and PRX5-overexpressing SNU-216 cells using TRI-Solution (Bio Science Technology, Seoul, Korea) according to the manufacturer's instructions. Complementary DNA was synthesized from $2 \mu \mathrm{g}$ of total RNA by using a Reverse Transcription Premix (Bioneer, Daejeon, Korea). PCR was performed using a PCR Premix (Bioneer). The following PCR primers were used: E-cadherin forward, 5'-TTGACGCCGAGAGCTACAC-3'; reverse, 5'-GTCGAC CGGTGCAATCTT-3'. Vimentin forward, 5'-TACAGGAA GCTGCTGGAAGG-3'; reverse, 5'-ACCAGAGGGAGTGAA TCCAG-3'. Snail forward, 5'-GCAACAAGGAATACCTC AGC-3'; reverse, 5'-TCTTGACATCTGAGTGGGTC-3'. GAPDH forward, 5'-ACCACAGTCCATGCCATCAC-3'; reverse, 5'-TCCACCACCCTGTTGCTGTA-3'.

Western blot analysis. Protein lysates were prepared using ice-cold PRO-PREP protein extraction solution (iNtRON Biotechnology Inc., Seongnam, Korea). Protein quantification was performed using an Infinite F50 microplate reader (Tecan Switzerland). For all samples, $20 \mu \mathrm{g}$ of the protein lysates were separated on a $10-15 \%$ SDS-polyacrylamide gel, transferred onto nitrocellulose membranes (Pall Corp., NY, USA), and blocked with 5\% skimmed milk (BD Biosciences, CA, USA). Overnight incubations with primary antibodies against $\beta$-actin (Santa Cruz Biotechnology Inc.), GAPDH (Cell Signaling Biotechnology, MA, USA), PRX5, vimentin (AbFrontier, Seoul, Korea), E-cadherin (BD Biosciences), Snail and Slug (both from Santa Cruz Biotechnology Inc.) were performed at $4^{\circ} \mathrm{C}$. The membranes were then washed 5 times with $10 \mathrm{mM}$ Tris- $\mathrm{HCl}(\mathrm{pH} 7.5)$ containing $150 \mathrm{mM} \mathrm{NaCl}$ and $0.1 \%$ Tween-20 (TBST). Finally, the membranes were incubated with horseradish peroxidaseconjugated goat anti-rabbit and anti-mouse antibodies (Thermo Fisher Scientific, IL, USA) for $2 \mathrm{~h}$ at room temperature. After washing off excess secondary antibodies, the membranes were washed 6 times with TBST. Specific binding was detected using Clarity Western ECL Substrate (Bio-Rad, CA, USA) according to the manufacturer's instructions.

Xenograft assay. Four-week-old male athymic nude mice (BALB/c-nu) were purchased from Central Lab. Animal Inc. (Seoul, Korea) and used according to the Animal Care and Use Guidelines of Kyungpook National University. When the mice were 5-weeks old, 5x10 ${ }^{6} \mathrm{SNU}-216$ and SNU-668 cells resuspended in $200 \mu \mathrm{l}$ phosphate-buffered saline (PBS) were subcutaneously injected using a 31-gauge needle. The sizes of the resulting tumors were measured using a caliper and calculated using the following formula: $\mathrm{W}$ (width) ${ }^{2} \mathrm{x} \mathrm{L}$ (length)/2. After we measured tumor mass during 50 days, the mice were sacrificed.

Foci formation. SNU-216 and SNU-216_ PRX5 cell lines were seeded on 6-well plates. Two weeks after seeding, cells were washed twice with PBS, fixed with $4 \%$ paraformaldehyde
(Sigma, St. Louis, MO, USA), and stained with $0.1 \%$ crystal violet (Sigma).

Proliferation assay and invasion assay. To measure cell proliferation, cells were seeded onto 96-well ImageLock plates (Essen Bioscience, Ann Arbor, MI, USA) at a density of $5 \times 10^{4}$ cells/well and incubated in DMEM with $10 \%$ normal FBS for 5 days. The plates were scanned on the IncuCyte imager (Essen Bioscience) and the data was analyzed via the IncuCyte Cell Proliferation assay software. Results are representative of three independent experiments. For the invasion assay, a scratch wound was generated using WoundMaker (Essen Bioscience), and the resulting cells were washed twice with PBS. The invasion assay was initiated by overlaying the cells with Matrigel (BD Biosciences) and images were acquired with IncuCyte Zoom (Essen Bioscience).

Statistical analysis. Quantitative data are presented as the mean \pm standard error of the mean (SEM) from at least three independent experiments. Comparisons between groups were analyzed using the unpaired two-tailed Student's t-test, or one-way or two-way ANOVA with post hoc analyses when appropriate, as indicated. Survival curves were visualized by applying Kaplan-Meier curves, and P-values were determined by the log-rank test, $\mathrm{P}<0.05$ was considered significant. Multivariate analysis of survival was used in a Cox proportional hazard regression model. All statistical analysis was conducted using SPSS 13.0 (SPSS Inc., Chicago, IL, USA).

\section{Results}

The expression of PRX5 is associated with poor prognosis of patients with gastric cancer. Between 2000 and 2003, we followed up 210 patients that underwent elective surgery for gastric cancer. The 5-year follow-up was conducted in two different groups. Patients with gastric cancer that showed PRX5 positive expression typically had a reduced 5-year survival of $36.8 \%$ compared to $58.7 \%$ of patients with gastric cancer that showed PRX5-negative expression, as depicted in the survival curve (log-rank test, $\mathrm{P}=0.007$; Fig. 1A). In addition, patients with high PRX5 expressing tumors showed a reduced 5-year survival of $22.7 \%$ compared to $52.4 \%$ for patients with low PRX5-expressing tumors (log-rank test, $\mathrm{P}=0.0004$; Fig. 1B). However, no significant correlation was found between PRX1-4 expression and changes in survival rates (Fig. 1C). Also, as expected, we estimated that no significant correlation between PRX1-4 expression levels and changes in 5-year survival rates. Overall, the 5-year survival data analyzed via the log-rank test indicated that overexpression of PRX5 is correlated with poor prognosis, and that this difference was statistically significant.

We also examined whether upregulation of PRX5 is linked to poor prognosis of gastric cancer, immunohistochemical analysis was performed in 210 paired gastric cancer tissues using PRX5 specific antibody. As shown in Fig. 2, PRX5 expression was mainly located in the membrane, cytoplasm, and nucleus of gastric cancer tissues. In the case of heterogeneous staining within the samples, tumor tissues were classified into four grades based on the staining intensity: PRX5 was weakly located in the cytoplasm, weak staining; PRX5 was located 

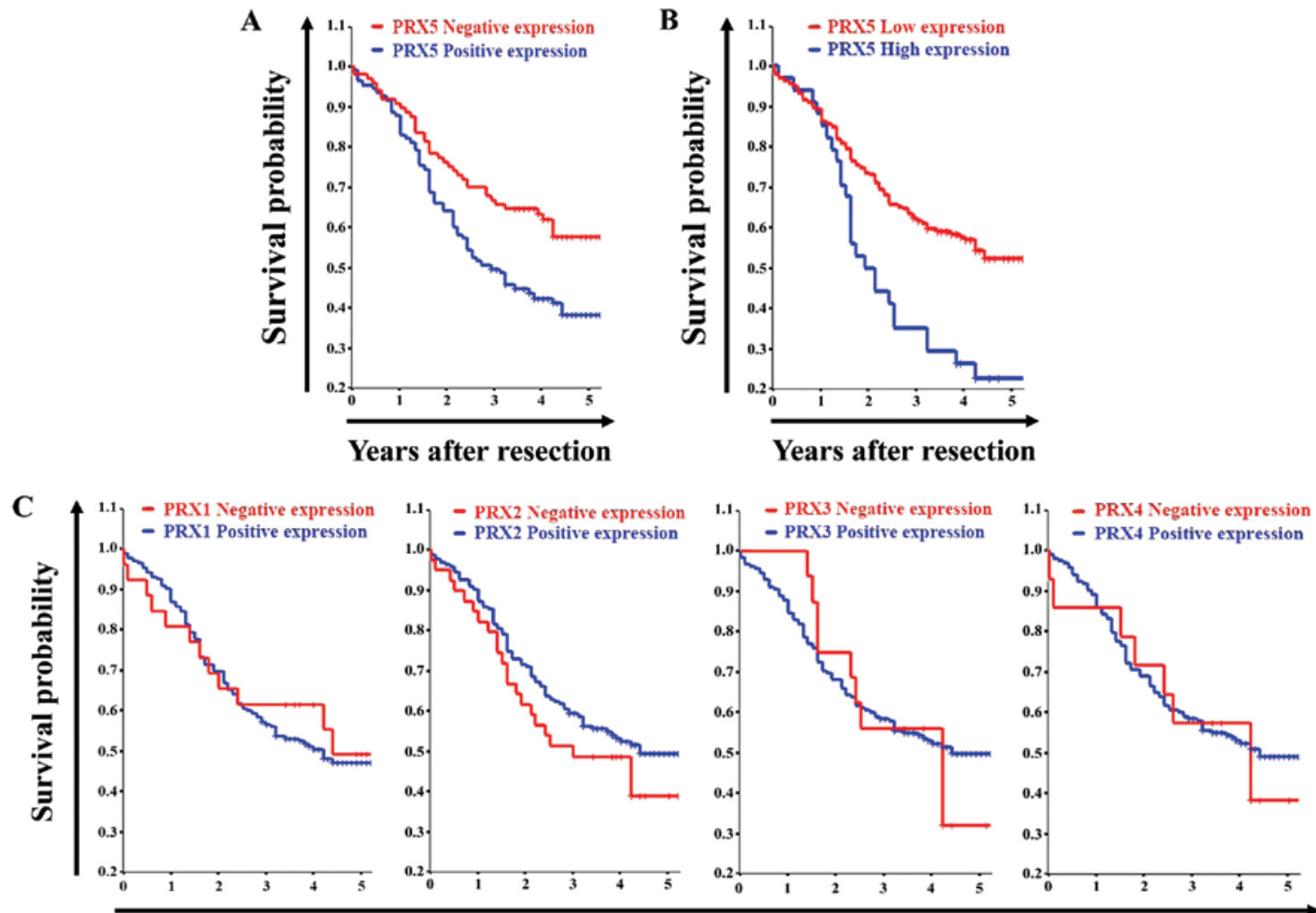

\section{Years after resection}

Figure 1. The 5-year survival rates of gastric cancer patients with expression of PRXs. (A) Positive and negative expression of PRX5 (P=0.007). (B) High and low expression of PRX5 ( $\mathrm{P}=0.0004)$. (C) Positive and negative expression of PRX1 ( $\mathrm{P}=0.73)$, $\mathrm{PRX} 2(\mathrm{P}=0.25), \mathrm{PRX} 3(\mathrm{P}=0.61), \mathrm{PRX} 4$ ( $\mathrm{P}=0.72)$. $\mathrm{P}-\mathrm{value}$ is statically significant in Chi-square test. Each PRX represents two groups: positive and negative expression group. Group comparisons of categorical variables were evaluated using the Chi-square test or linear-by-linear association. T stage, T1/T2/T3/T4; TNM (tumor-node-metastasis staging), stage I/stage II/stage III/ stage IV. P-value is statically significant in Chi-square test.

A

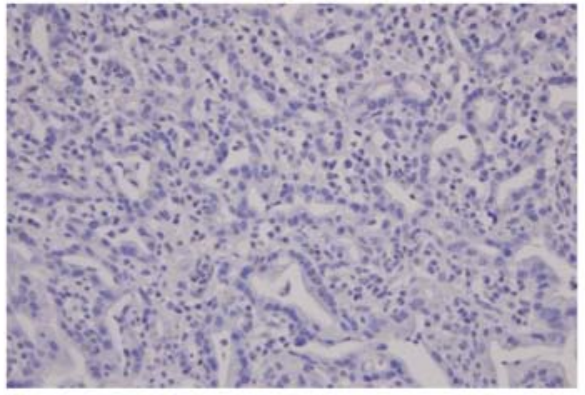

C

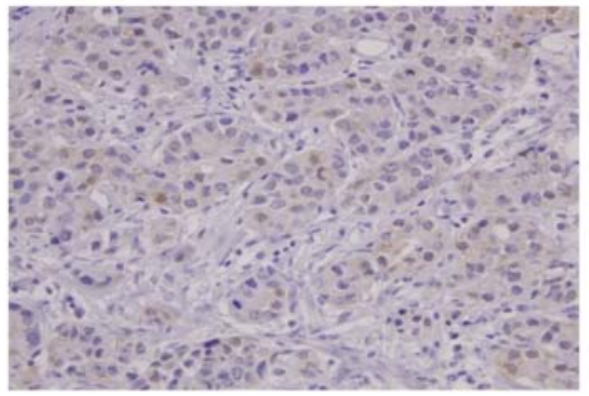

B

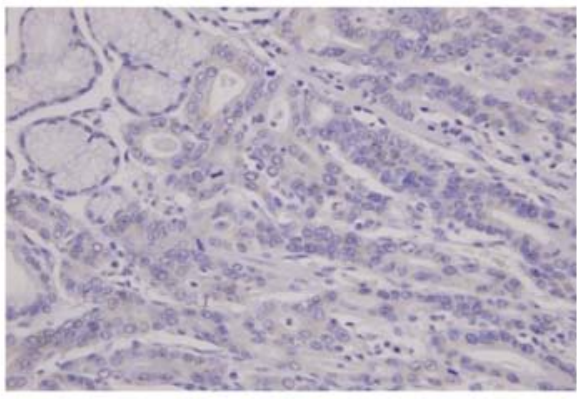

D

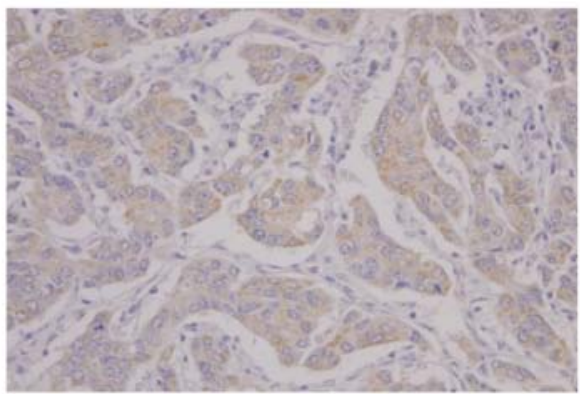

Figure 2. Representative photomicrographs of immunohistochemical staining for PRX5 in human gastric cancer tissues. PRX5 was expressed mainly in the cytoplasm of cancer cells. It was also focally expressed in the nuclei of some cancer cells. (A) No staining intensity. (B) Weak staining intensity. (C) Intermediate staining intensity. (D) Strong staining intensity (x400).

in the membrane and cytoplasm, intermediate staining; PRX5 was strongly located in the membrane, cytoplasm, and nucleus, strong staining. These data suggested that PRX5 is a potential factor that may contribute to poor prognosis of gastric cancer. The relationships between PRX5 expression and prognostic factors, such as expression levels of PRX5 and tumor size and 
Table I. Multivariate analysis of the prognostic factors in patient survival.

\begin{tabular}{lcc}
\hline Factors & HR $(95 \% \mathrm{CI})$ & P-value \\
\hline $\begin{array}{l}\text { Tumor size } \\
(>5 \mathrm{~cm} \text { vs. } \leq 5 \mathrm{~cm})\end{array}$ & $2.171(1.186-3.977)$ & $<0.01$ \\
$\begin{array}{l}\text { Stage (III, IV vs I, II) } \\
\begin{array}{l}\text { PRX\% expression } \\
\text { positive vs. negative) }\end{array}\end{array}$ & $1.528(0.875-2.468-8.214)$ & $<0.01$ \\
\hline
\end{tabular}

grade are summarized in Table $\mathrm{I}$. The prognostic factors in patient' survival were tumor size $(\mathrm{P}=0.001)$, high expression of PRX5 ( $\mathrm{P}=0.001)$ and later tumor stage III/IV ( $\mathrm{P}<0.001$; Table I). The difference was statistically significant.

Correlation of PRX5 expression and clinicopathological parameters. The relationship between PRX5 expression and clinicopathological parameters, such as tumor size, differentiation status, invasion and tumor grade are summarized in Table II. No significant associations with gender and age were observed with the clinicopathological parameters analyzed including clinical stages and lymph node metastasis. Furthermore, there were no significant differences in tumor location, lymphatic invasion, venous invasion, and proportion of tumor size $>5 \mathrm{~cm}$, depth of tumor and TNM stages in PRX1-4 expressing tumors. However, positive expression of PRX3 and PRX4 tend to be associated with more aggressive tumor differentiation (PRX3; $\mathrm{P}=0.02$, $\mathrm{PRX} 4 ; \mathrm{P}=0.01$ ). In addition, expression of PRX5 was significantly correlated with tumor size ( $\mathrm{P}=0.001)$, depth of tumor ( $\mathrm{T}$ status; $\mathrm{P}=0.001$ ), lymph node involvement ( $\mathrm{N}$ status; $\mathrm{P}=0.03$ ), and $\mathrm{TNM}^{*}$ stage $(\mathrm{P}=0.003)$. Finally, high expression of PRX5 was associated with lymphatic invasion (PRX5: $\mathrm{P}=0.03$; Table II). These data are consistent with the hypothesis that PRX5 is a potential factor that may contribute to the poor prognosis of gastric cancer.

High expression of PRX5 is induced to mesenchymal phenotype in gastric cancer. Recently, increasing evidence suggests that EMT may also contribute to poor prognosis in patients with gastric cancer $(25,26)$. EMT-related markers are significantly expressed and can also act as prognostic factors (5-8). The 5-year survival rate of patients suggests that tumors positive for PRX5 are associated with a lower survival probability. Thus, we decided to focus on studying the relationship between PRX5 and EMT. First, we estimated the expression of PRX5 in various gastric cancer cell lines. SNU-216 cells showed the lowest expression of PRX5 whereas SNU-668 cells showed the highest expression of PRX5 (data not shown). We then analyzed EMT in these cell lines, which is a requirement for several molecular expression changes. Vimentin is regulated to change the cell adhesion and migration capacity in cancer cells, and loss of E-cadherin is required for tumor invasion. Thus, expression changes in vimentin and E-cadherin are typical features of EMT $(6-8,16,17)$. We determined the molecular level of vimentin and E-cadherin. Interestingly, both RT-PCR and western blot analysis showed high levels of vimentin and low levels of E-cadherin in SNU-668 cells expressing high levels of endogenous PRX5, which was not observed in SNU-216 cells expressing low levels of endogenous PRX5 (Fig. 3A and B). To investigate whether PRX5 influenced tumor growth in vivo, we performed the tumor xenograft assay. Tumor size was measured $\sim 50$ days after the gastric cancer cell injection. SNU-668 cells gave rise to larger tumors than the SNU-216 cells (Fig. 3C). This suggests that PRX5 may contribute to the mesenchymal phenotype in gastric cancer.

Exogenous overexpression of PRX5 aggravates carcinogenicity in gastric cancer cells. To investigate the functional effect of PRX5 in carcinogenesis, we overexpressed V5-tagged PRX5 (PRX5-V5) in SNU-216 cells (SNU-216_PRX5) which was low expressing levels of endogenous PRX5. SNU-216 PRX5 was then confirmed via western blotting (Fig. 4A). To analyze the carcinogenic capacity between SNU-216 cells and SNU-216_PRX5 cells, we performed the foci formation assay and invasion assay to assess their proliferation. The number of foci was increased in SNU-216_PRX5 cells compared with SNU-216 cells (Fig. 4B). Moreover, we observed higher proliferation rate in SNU-216_PRX5 cells than in SNU-216 cells. The proliferation rate of SNU-216_PRX5 cells was also faster than SNU-216 cells (Fig. 4C). Moreover, cancer cell invasion

Table II. Clinicopathological features of patients with gastric carcinoma organized by PRX1-5 expression.

\begin{tabular}{llllll}
\hline Clinicopathological features & PRX1 & PRX2 & PRX3 & PRX4 & PRX5 \\
\hline Sex (female/male) & 0.82 & 0.62 & 0.85 & 0.06 & 0.31 \\
Location & 0.61 & 0.73 & 0.41 & 0.31 & 0.98 \\
Tumor size $(>5 \mathrm{~cm} / \leq 5 \mathrm{~cm})$ & 0.09 & 0.67 & 0.85 & 0.53 & $<0.01$ \\
Differentiation (differentiated/undifferentiated) & 0.59 & 0.053 & 0.02 & 0.01 & 0.46 \\
Lymphatic invasion (yes/no) & 0.65 & 0.96 & 0.1 & 0.14 & 0.07 \\
Venous invasion (yes/no) & 0.97 & 0.71 & 0.67 & 0.14 & 0.24 \\
T stage (T3, 4/T1, 2) & 0.12 & 0.48 & 0.97 & 0.34 & $<0.01$ \\
Lymph node involvement (yes/no) & 0.32 & 0.85 & 0.18 & 0.69 & $<0.01$ \\
TNM stage (III, IV vs I, II) & 0.87 & 0.09 & 0.66 & 0.43 & $<0.01$ \\
\hline
\end{tabular}


A
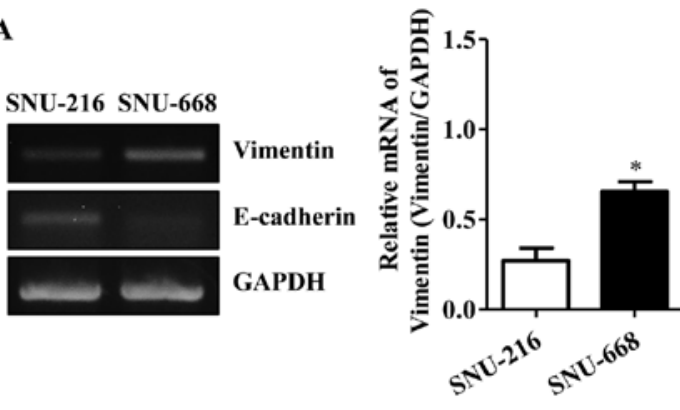
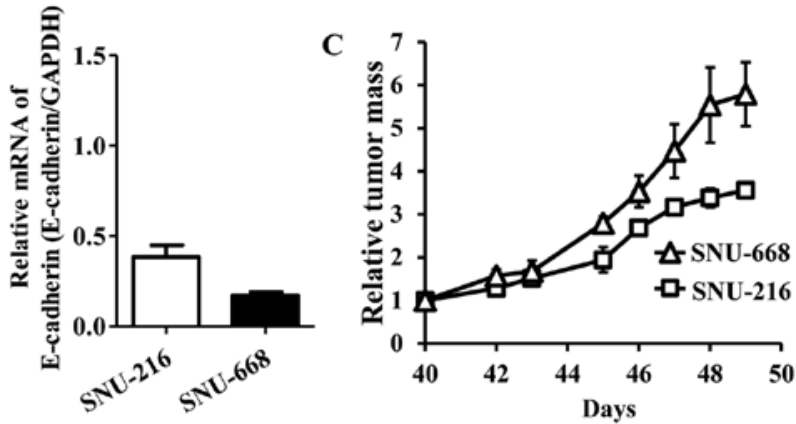

B
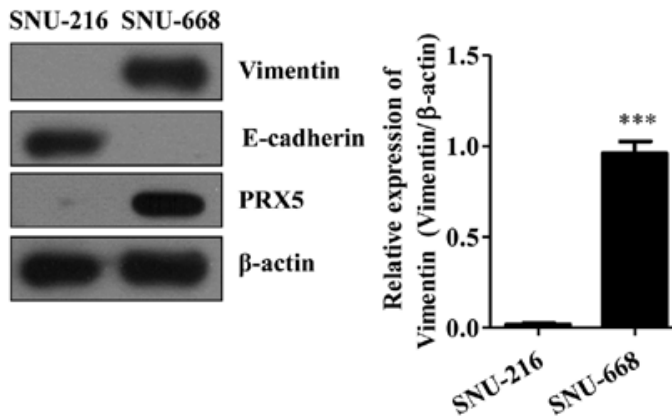
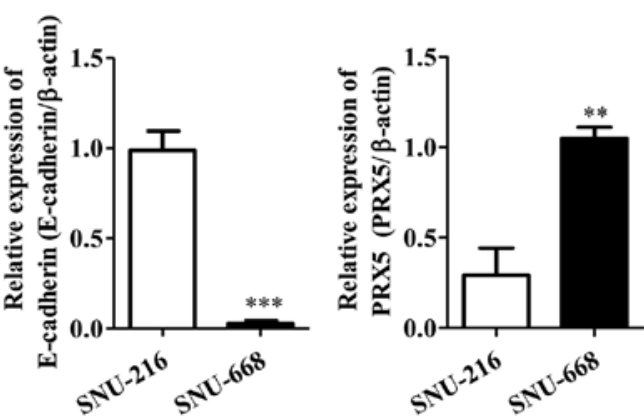

Figure 3. PRX5 is associated with mesenchymal phenotype and tumorigenicity in human gastric cancer cell line. (A) The mRNA levels of vimentin and E-cadherin in SNU-216 and SNU-668 human gastric cancer cell lines. The mRNA level was analyzed via RT-PCR. GAPDH was used as a control. (B) The protein expression levels of vimentin, E-cadherin, and PRX5 in SNU-216 and SNU-668 human gastric cancer cell lines. $\beta$-actin was used as a control. (C) Relative tumor volumes measured on the indicated days. Data are expressed as mean \pm SEM of 3 independent experiments $\left({ }^{*} \mathrm{P}<0.05,{ }^{* *} \mathrm{P}<0.01,{ }^{* * *} \mathrm{P}<0.001\right)$.

A

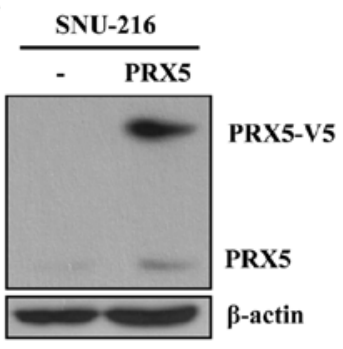

C

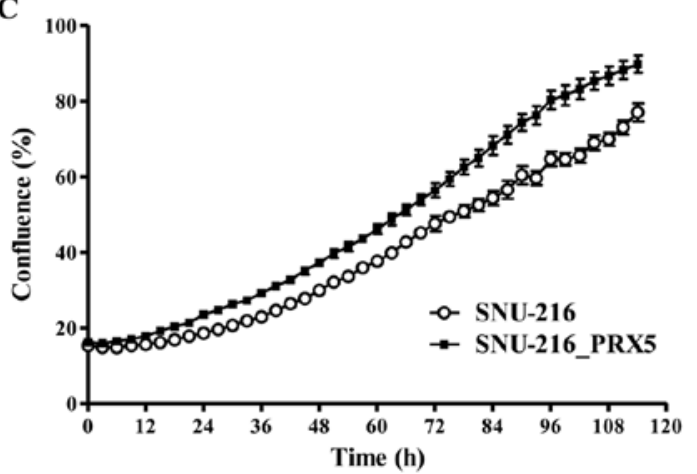

B

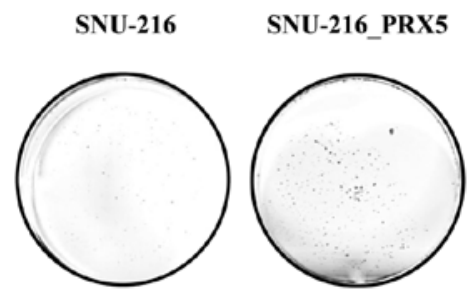

D

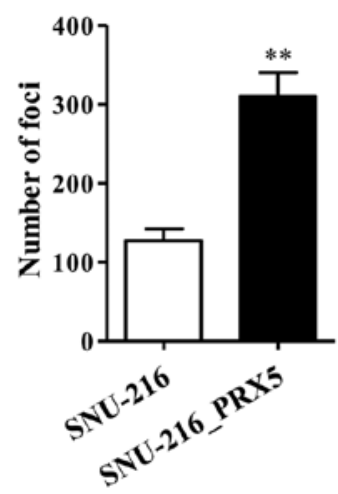

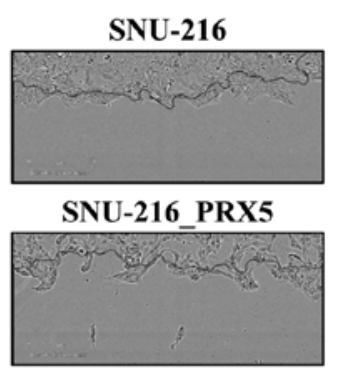

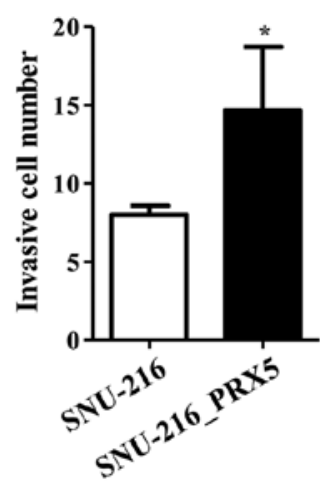

Figure 4. PRX5 intensified tumorigenicity. (A) The protein expression level of PRX5 in SNU-216 and V5-tagged PRX5-overexpressing SNU-216 cells (SNU-216_PRX5). $\beta$-actin was used as a control. (B) To confirm the role of PRX5 in SNU-216 and SNU-216_PRX5 cells, each line was cultured in 6-well plate with a seeding density of $1 \times 10^{4}$ cells. After 7 days, cells were stained with crystal violet. The graph on the right depicts the number of foci observed. (C) For the cell proliferation assay, the same number of SNU-216 and SNU-216_PRX5 cells were incubated in IncuCyte, which was also used to determine cell confluence. (D) The invasion assay was performed via IncuCyte migration kit. Data are expressed as mean \pm SEM of 3 independent experiments ("P $<0.05$, $\left.{ }^{* * *} \mathrm{P}<0.01,{ }^{* * * *} \mathrm{P}<0.001\right)$. 
A

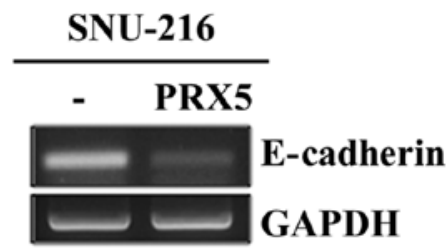

B

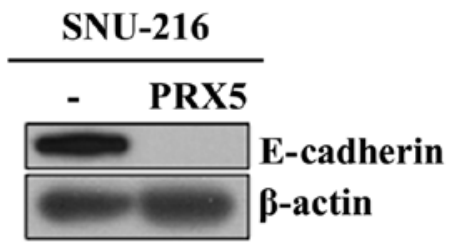

C

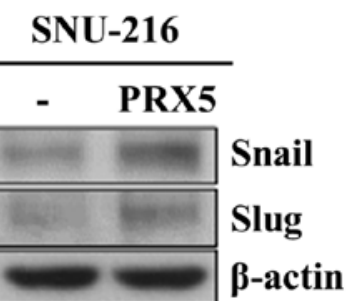

Figure 5.PRX5 decreases E-cadherin via upregulation of Snail.(A) The mRNA level of E-cadherin was confirmed by RT-PCR in SNU-216 and SNU-216_PRX5 cells. GAPDH was used as a control. (B) The protein expression levels of E-cadherin in SNU-216 and SNU-216_PRX5 cells were examined using western blotting. $\beta$-actin was used as a control. (C) The protein expression of Snail and Slug was examined by western blotting in SNU-216 and SNU-216_PRX5. $\beta$-actin was used as control.

was also higher in SNU-216_PRX5 cells than in SNU-216 cells (Fig. 4D). Therefore, these results suggest that overexpression of PRX5 enhanced carcinogenicity by increasing the proliferation and invasiveness of gastric cancer cells.

EMT may be enhanced by PRX5 in gastric cancer cells. Downregulation of E-cadherin is considered an important step during EMT. E-cadherin also acts as a suppressor during tumor progression $(16,17)$. Thus, we examined the mRNA and protein levels of E-cadherin in SNU-216_PRX5 cells. As expected, the mRNA levels of E-cadherin was decreased (Fig. 5A). Moreover, the protein level of E-cadherin was also declined (Fig. 5B). We also investigated how PRX5 may be regulating E-cadherin expression by analyzing the expression level of Snail, a known E-cadherin repressor (7). As expected, the protein levels of Snail and Slug increased in SNU-216_PRX5 cells (Fig. 5C). As a result, we speculated that PRX5 may upregulate Snail and Slug, consequently resulting in downregulation of E-cadherin.

\section{Discussion}

In this study, we investigated the correlation between PRX5 and carcinogenesis in gastric cancer cells. We noted that the 5-year survival rates of patients with PRX5-positive expressing gastric cancer tumors are poorer than the survival rates of those with PRX5-negative expressing tumors (log-rank test, $\mathrm{P}=0.007$; Fig. 1A). We also showed that the overexpressionof PRX5 is related to poor prognosis in patients with gastric cancer. To show the correlation between PRX5 and carcinogenesis, we analyzed two gastric cancer cell lines. The highest expression of PRX5 (SNU-668) and the lowest expression of PRX5 (SNU-216) gastric cancer cell line were selected, because we wanted to confirm precise difference between low and high expression of PRX5 gastric cancer cell lines (data not shown). We observed that tumor growth of SNU-668 was faster than that of SNU-216 (Fig. 3C). Moreover, exogenous expression of PRX5 in SNU-216 cells induced cell proliferation, invasion, and foci formation (Fig. 4). These results suggested that high level of PRX5 expression might enhance the tumorigenic phenotype in gastric cancer cells.

Previous studies have shown that EMT is related to cancer progression and metastasis (25,27-29). Changes in expression are also observed in several molecular markers for EMT such as E-cadherin and vimentin, as well as transcription factors such as Snail (6-8). These changes result in a poorer prognosis for patients with gastric cancer. Vimentin is an important factor of mesenchymal cells and is critically involved in cell-cell adhesion, migration and EMT-related signaling pathways. Vimentin also cooperates with membrane integrins to promote the mesenchymal architecture. Therefore, vimentin expression correlates with tumor progression and the survival rate of patients with gastric cancer $(16,17)$. E-cadherin plays a critical role in cell-cell adhesion and the maintenance of cell polarity in epithelial cells. The loss of E-cadherin is associated with cancer cell invasion and worse survival (19,21-23). In addition, several reports even suggest that regulation of EMT may be the key to curing cancer (28-31). In the present study, we investigated how PRX5 may be regulating tumorigenicity of gastric cancer cells by analyzing correlations between PRX5 expression and EMT markers. First, we confirmed mRNA and protein level of E-cadherin and vimentin in both SNU-216 and SNU-668 cells (Fig. 3A and B). SNU-216 cells showed higher levels of E-cadherin and lower levels of vimentin, whereas SNU-668 cells showed lower levels of E-cadherin and higher levels of vimentin. In addition, overexpression of PRX5 in SNU-216 cells resulted in a significant reduction of E-cadherin (Fig. 5A and B). Furthermore, to investigate how PRX5 regulates E-cadherin expression, we analyzed the protein expression level of Snail and Slug, a well-known repressor of E-cadherin (7). Snail and Slug expression were significantly increased in SNU-216_PRX5 (Fig. 5C). Our data indicated that PRX5 enhanced the expression of Snail and Slug by which the expression of E-cadherin was subsequently repressed. Thus our these data suggest that overexpression of PRX5 significantly correlated with poorer prognosis in gastric cancer.

Furthermore, previous studies have also suggested that reactive oxygen species (ROS) and reactive nitrogen species (RNS) are generated in high amounts during cancer development. Thus, factors involved in these processes may provide useful candidate diagnostic and prognostic markers for many types of cancers (2). Excessive ROS and RNS may also enhance damaging reactions within lipids, proteins, and DNA, which can then lead to tumor progression (12-15). Normally, certain signaling pathways are activated to control ROS and RNS concentrations within the cell. These signaling pathways include enzymes such as catalase, superoxide dismutase, and glutathione peroxidase, as well as other factors such as antioxidants and reducing agents (15). A large number of researchers 
have reported that $\mathrm{PRXs}$ is an antioxidant peroxidase protecting against ROS and ROS-induced damage $(14,15,18)$. However, other studies have also suggested that PRXs may significantly enhance tumorigenic efficacy and the metastatic capability of various cancer cells independent on ROS (19-23).

Finally, PRX5 has been reported to be the type of PRX family that reacts with both hydrogen peroxide and peroxynitrite $(19,20)$. To date, six isoforms of PRXs (PRX1-6) have been identified in mammalian tissues. Also, they are divided into three major subclasses: typical 2-cysteine (PRX1-4), atypical 2-cysteine PRX (PRX5), and 1-cysteine PRX (PRX6) (10). These isoenzymes are widely distributed throughout several subcellular structures, including the mitochondria, peroxisomes, ER, protoplasm, and the cell membrane (9). PRX family proteins are also upregulated in many types of tumors. For instance, PRX1-6 are upregulated in breast, lung and malignant mesothelioma cancer (21-23). PRX1, 2, and 6 are upregulated in ovarian cancer tissues, and PRX2-4 are upregulated in prostate cancer tissues $(14,15)$. In breast cancer, PRX5 is associated with a larger tumor size, positive lymph nodes, and TNM classification (16). In colorectal cancer, PRX1, -2 and -5 are associated with an advanced stage of cancer and lymph node metastasis (30). Overall, the PRX family of proteins have been linked to tumor development and progression $(19,21-23,30)$. In the present study, high expression of PRX5 was significantly correlated with tumor cell proliferation, tumor size, foci formation, invasion, lymph node metastasis, and advanced TNM classification. Patients with higher levels of PRX5 expression in gastric cancer cells also showed a shorter 5-year survival rate. In the Cox hazard regression covariate analysis, expression of PRX5 was shown to have an impact on survival.

Taken together, PRX5 is implicated in multifunctional mechanisms that promote EMT and tumorigenic phenotype in gastric cancer cells. In addition, PRX5 is an important factor of diagnosis that may contribute to poor prognosis. Finally, PRX5 is a putative therapeutic target and clinical strategy for various cancers overexpressing PRX5.

\section{Acknowledgements}

This study was supported by a grant from the Korean Health Technology R\&D Project, Ministry of Health \& Welfare (HI15C0789), by a Chungnam National University Hospital Research Fund, 2012, grants from the KRIBB Research Initiative Program (KGM4611714), and the National Research Foundation of Korea funded by the Republic of Korea Government (NRF-2014R1A2A1A11054095 and NRF-2017R1A2B4008176).

\section{References}

1. Yoon JH, Choi WS, Kim O, Choi BJ, Nam SW, Lee JY and Park WS: Gastrokine 1 inhibits gastric cancer cell migration and invasion by downregulating RhoA expression. Gastric Cancer 20: 274-285, 2017.

2. Burlaka AP, Ganusevich II, Gafurov MR, Lukin SM and Sidorik EP: Stomach Cancer: interconnection between the redox state, activity of MMP-2, MMP-9 and stage of tumor growth. Cancer Microenviron 9: 27-32, 2016.

3. Kim KH, Lee KW, Baek SK, Chang HJ, Kim YJ, Park DJ, Kim JH, Kim HH and Lee JS: Survival benefit of gastrectomy \pm metastasectomy in patients with metastatic gastric cancer receiving chemotherapy. Gastric Cancer 14: 130-138, 2011.
4. Cheon SH, Rha SY, Jeung HC, Im CK, Kim SH, Kim HR, Ahn JB, Roh JK, Noh SH and Chung HC: Survival benefit of combined curative resection of the stomach (D2 resection) and liver in gastric cancer patients with liver metastases. Ann Oncol 19: 1146-1153, 2008.

5. Kalluri R and Weinberg RA: The basics of epithelial-mesenchymal transition. J Clin Invest 119: 1420-1428, 2009.

6. Otsuki S, Inokuchi M, Enjoji M, Ishikawa T, Takagi Y, Kato K, Yamada H, Kojima K and Sugihara K: Vimentin expression is associated with decreased survival in gastric cancer. Oncol Rep 25: 1235-1242, 2011.

7. Rosivatz E, Becker KF, Kremmer E, Schott C, Blechschmidt K, Höfler $\mathrm{H}$ and Sarbia M: Expression and nuclear localization of Snail, an E-cadherin repressor, in adenocarcinomas of the upper gastrointestinal tract. Virchows Arch 448: 277-287, 2006.

8. Thiery JP, Acloque H, Huang RY and Nieto MA: Epithelialmesenchymal transitions in development and disease. Cell 139: 871-890, 2009.

9. Halliwell B, Gutteridge JM and Cross CE: Free radicals, antioxidants, and human disease: Where are we now? J Lab Clin Med 119: 598-620, 1992.

10. Finkel T and Holbrook NJ: Oxidants, oxidative stress and the biology of ageing. Nature 408: 239-247, 2000.

11. Finkel T: Oxidant signals and oxidative stress. Curr Opin Cell Biol 15: 247-254, 2003.

12. Grek CL and Tew KD: Redox metabolism and malignancy. Curr Opin Pharmacol 10: 362-368, 2010.

13. Poli G, Leonarduzzi G, Biasi F and Chiarpotto E: Oxidative stress and cell signalling. Curr Med Chem 11: 1163-1182, 2004.

14. Tehan L, Taparra K and Phelan S: Peroxiredoxin overexpression in MCF-7 breast cancer cells and regulation by cell proliferation and oxidative stress. Cancer Invest 31: 374-384, 2013.

15. Kropotov A, Gogvadze V, Shupliakov O, Tomilin N, Serikov VB, Tomilin NV and Zhivotovsky B: Peroxiredoxin V is essential for protection against apoptosis in human lung carcinoma cells. Exp Cell Res 312: 2806-2815, 2006.

16. Caldeira J, Figueiredo J, Brás-Pereira C, Carneiro P, Moreira AM, Pinto MT, Relvas JB, Carneiro F, Barbosa M, Casares F, et al: E-cadherin-defective gastric cancer cells depend on Laminin to survive and invade. Hum Mol Genet 24: 5891-5900, 2015.

17. Chen HC, Chu RY, Hsu PN, Hsu PI, Lu JY, Lai KH, Tseng HH, Chou NH, Huang MS, Tseng CJ, et al: Loss of E-cadherin expression correlates with poor differentiation and invasion into adjacent organs in gastric adenocarcinomas. Cancer Lett 201: 97-106, 2003

18. Tölle A, Schlame M, Charlier N, Guthmann F and Rüstow B: Vitamin E differentially regulates the expression of peroxiredoxin-1 and -6 in alveolar type II cells. Free Radic Biol Med 38: 1401-1408, 2005.

19. Kim B, Park J, Chang KT and Lee DS: Peroxiredoxin 5 prevents amyloid-beta oligomer-induced neuronal cell death by inhibiting ERK-Drp1-mediated mitochondrial fragmentation. Free Radic Biol Med 90: 184-194, 2016.

20. Knoops B, Goemaere J, Van der Eecken V and Declercq JP: Peroxiredoxin 5: Structure, mechanism, and function of the mammalian atypical 2-Cys peroxiredoxin. Antioxid Redox Signal 15: 817-829, 2011.

21. Li L, Zhang YG and Chen CL: Anti-apoptotic role of peroxiredoxin III in cervical cancer cells. FEBS Open Bio 3: 51-54, 2012.

22. Whitaker HC, Patel D, Howat WJ, Warren AY, Kay JD, Sangan T, Marioni JC, Mitchell J, Aldridge S, Luxton HJ, et al: Peroxiredoxin- 3 is overexpressed in prostate cancer and promotes cancer cell survival by protecting cells from oxidative stress. Br J Cancer 109: 983-993, 2013.

23. Choi H, Chang JW and Jung YK: Peroxiredoxin 6 interferes with TRAIL-induced death-inducing signaling complex formation by binding to death effector domain caspase. Cell Death Differ 18: 405-414, 2011.

24. Japanese Gastric Cancer Association: Japanese classification of gastric carcinoma. 2nd English edition. Gastric Cancer 1: 10-24, 1998.

25. Han G, Lu SL, Li AG, He W, Corless CL, Kulesz-Martin M and Wang XJ: Distinct mechanisms of TGF-beta1-mediated epithelial-to-mesenchymal transition and metastasis during skin carcinogenesis. J Clin Invest 115: 1714-1723, 2005.

26. Lee JM, Dedhar S, Kalluri R and Thompson EW: The epithelialmesenchymal transition: New insights in signaling, development, and disease. J Cell Biol 172: 973-981, 2006.

27. Shih JY and Yang PC: The EMT regulator slug and lung carcinogenesis. Carcinogenesis 32: 1299-1304, 2011. 
28. Iwatsuki M, Mimori K, Yokobori T, Ishi H, Beppu T, Nakamori S, Baba $\mathrm{H}$ and Mori M: Epithelial-mesenchymal transition in cancer development and its clinical significance. Cancer Sci 101: 293-299, 2010.

29. Sung WJ, Park KS, Kwak SG, Hyun DS, Jang JS and Park KK: Epithelial-mesenchymal transition in patients of pulmonary adenocarcinoma: Correlation with cancer stem cell markers and prognosis. Int J Clin Exp Pathol 8: 8997-9009, 2015.

30. Zhai X, Zhu H, Wang W, Zhang S, Zhang Y and Mao G: Abnormal expression of EMT-related proteins, S100A4, vimentin and E-cadherin, is correlated with clinicopathological features and prognosis in HCC. Med Oncol 31: 970, 2014.
31. Han MW, Lee JC, Kim YM, Cha HJ, Roh JL, Choi SH, Nam SY, Cho KJ, Kim SW and Kim SY: Epithelial-mesenchymal transition: Clinical implications for nodal metastasis and prognosis of tongue cancer. Otolaryngol Head Neck Surg 152: 80-86, 2015. 